\title{
Indirect comparisons of efficacy and safety between seven newer targeted agents for metastatic renal cell carcinoma: A network meta-analysis of randomised clinical trials
}

\author{
HENRY W.C. LEUNG ${ }^{1,2}$, AGNES L.F. CHAN ${ }^{2,3}$ and SHUN-JEN LIN ${ }^{4}$ \\ ${ }^{1}$ Department of Radiation Oncology, Tainan Municipal An-Nan Hospital-China Medical University, Tainan 709; \\ ${ }^{2}$ Hsin Sheng College of Medicine Care and Management, Taoyuan 325; ${ }^{3}$ Department of Pharmacy, \\ Tainan Municipal An-Nan Hospital-China Medical University, Tainan 709; \\ ${ }^{4}$ School of Pharmacy, Kaohsiung Medical University, Kaohsiung 807, Taiwan
}

Received January 15, 2014; Accepted February 26, 2014

DOI: $10.3892 / \mathrm{mco} .2014 .323$

\begin{abstract}
This network meta-analysis aimed to compare the clinical efficacy and safety among 7 newer targeted agents for the treatment of metastatic renal cell carcinoma (mRCC). All randomised clinical trials (RCTs) of targeted therapeutic drugs for mRCC were included. The study selection, data extraction and quality assessment were performed independently by two reviewers. The analysis evaluated efficacy outcomes [improvement in the median progression-free survival (PFS)] and safety outcomes (number of withdrawals due to adverse events). The network analysis included direct and indirect analyses. The quality of the selected studies was assessed using the Grading of Recommendations, Assessment, Development and Evaluations (GRADE) method. We identified 7 articles eligible for inclusion in the study. The direct comparison of the targeted agents indicated better efficacy in terms of longer PFS, but worse safety (more withdrawals due to adverse events). The indirect analysis demonstrated that axitinib was significantly more effective compared to panzopanib; sunitinib was superior to sorafenib and temsirolimus regarding efficacy outcome, without any statistically significant difference in the safety outcome. The results of the quality assessment indicated moderate scores using the GRADE method. In conclusion, the result of this network analysis suggested that sunitinib and axitinib may be more clinically efficient and axitinib is associated with
\end{abstract}

Correspondence to: Dr Agnes L.F. Chan, Department of Pharmacy, Tainan Municipal An-Nan Hospital-China Medical University, No 66, Sec. 2, Changhe Road, Tainan 709, Taiwan E-mail: agnes.lf@gmail.com

Key words: mixed treatment comparisons, targeted therapy, progression-free survival, hazard ratio, serious adverse event, metastatic renal cell carcinoma, indirect treatment comparisons, GPU-Enabled Many-Task Computing a lower risk of adverse events compared to sorafenib, pazopanib and temsirolimus.

\section{Introduction}

Kidney cancer is the 13th most common malignancy, with $\sim 271,000$ new cases diagnosed worldwide in 2008 (1). The incidence rates of kidney cancer are the highest in Europe, North America and Australia, whereas they are low in India, Japan, Africa and China. Over the past several decades, the incidence rates of kidney cancer have been steadily increasing by $\sim 1.7 \%$ in males and $2.2 \%$ in females each year in the United States $(2,3)$. In 2010, 58,240 new cases of kidney cancer and 13,040 deaths were reported in the United States (4), accounting for $\sim 4 \%$ of all new primary cancer cases. In Taiwan, the estimated annual incidence rate of kidney cancer was 5.22 and 2.45 per 100,000 males and females, respectively, with estimated mortality rates of 2.56 and 2.02 per 100,000 males and females, respectively, in 2008 (5).

Approximately 25-30\% of patients with kidney cancer present with metastases at the time of diagnosis, since the majority of kidney cancer cases initially present with an asymptomatic clinical course. Patients with localized renal cell carcinoma (RCC) may exhibit longer times to relapse following nephrectomy, with a median duration of 15-18 months. The majority of relapses occur within the first 3 years after nephrectomy $(6,7)$. Metastatic RCC (mRCC) is a major clinical problem for oncologic health care worldwide, due to its extremely poor prognosis (6).

The economic impact of RCC on healthcare resources has increasing significantly with its increasing incidence in the United States and Asian countries. The annual economic burden of RCC was estimated to be 0.60-5.19 billion USD (16,488-43,805 USD per patient) in the United States (8). Furthermore, the economic burden of RCC is expected to grow with the increasing use of targeted therapies (9).

New targeted agents for the treatment of RCC are continuously developed since 2005 . There are currently two groups of such agents, vascular endothelial growth factor-targeted therapies and mammalian target of rapamycin inhibitors. 
Seven newer targeted agents (sorafenib, sunitinib, pazopanib, everolimus, temsirolimus, axitinib and cediranib) have been approved by the Food and Drug Administration of the United States and Taiwan for the treatment of RCC. However, these agents are significantly more costly compared to traditional chemotherapy. In particular, due to the lack of head-to-head comparisons, the information regarding their comparative effectiveness is limited and their toxicities are a major concern. Hence, with the aid of network meta-analysis, this study aimed to compare the efficacy and safety among these 7 newer targeted agents for patients with $\mathrm{mRCC}$.

\section{Materials and methods}

Literature search. A systematic literature search of the Medline, Embase, Cochrane Library and ClinicalTrials. gov databases was performed to identify all clinical studies on those 7 newer targeted agents for the treatment of $\mathrm{mRCC}$ between January 1, 1999 and August 31, 2013. The databases were searched using the following medical subject headings or text keywords: metastatic or advanced renal cell cancer or carcinoma and targeted agents. In addition, we searched the references of the selected studies manually to identify any overlooked literature. Only randomised clinical trials (RCTs) of the 7 targeted agents for $\mathrm{mRCC}$ published in English were considered in this study.

Data extraction. Two reviewers screened the titles and abstracts of all identified studies to exclude ineligible studies and then read the full texts of the remaining studies independently. We collected data on patient demographic and clinical characteristics, study design, interventions, direct outcomes and sample sizes from each of the selected studies using a standardised data-recording form.

Quality assessment. We used the Cochrane Collaboration Recommendations for Assessment to evaluate the risk of bias in each included study (10). The criteria for measuring the risk of bias included i) allocation concealment, ii) random sequence generation, iii) presence of blinding in the studies (patients, assessors and physicians), iv) incomplete outcome data, v) selective outcome reporting and vi) other sources of bias. Each study was evaluated explicitly based on these 6 criteria using the following scoring system: low risk of bias, high risk of bias or unclear (either lack of information or uncertainty regarding the potential for bias). We also assessed the quality of evidence in these studies using the Grading of Recommendations, Assessment, Development and Evaluations (GRADE) profiler (GRADEpro) software, version 3.2 (The Cochrane Information Management System) (11). This software was used to perform several of the required calculations, guide us through the process of grading the quality of the evidence and create a Summary of Findings table (10).

Data synthesis. We conducted a network meta-analysis, referred to as mixed or multiple treatment comparisons (MTC) meta-analysis, to compare the outcomes among the 7 newer targeted agents for $\mathrm{mRCC}$, which included direct (i.e., head-to-head) and indirect treatment comparisons $(12,13)$. The two major studied outcomes for comparison were i) efficacy, in terms of longer progression-free survival (PFS) as measured by the hazard ratio (HR) and ii) safety, defined by the withdrawals due to adverse events, as measured by the odds ratio. Indirect treatment comparisons were usually performed based on the available direct treatment comparisons with common comparators, such as placebo or a standard treatment.

Direct (head-to-head) comparisons of treatment effects. We summarized the results of the available direct (head-to-head) treatment comparisons from the included 7 RCTs using the Review Manager (RevMan) software, version 5.2.3 (The Cochrane Collaboration, Copenhagen, Denmark) (14).

Indirect comparisons of treatment effects. Two methods of indirect meta-analysis were used: the most frequently used method was the adjusted indirect comparisons using the random effects model as described by Bucher et al (15) and Caldwell et al (16) and the Bayesian method outlined by $\mathrm{Lu}$ and Ades (13).

According to the Bucher method, we calculated the logarithm of the HR and its standard error and used each in indirect comparisons for each included RCT. These comparisons included direct within-trial comparisons between two treatment strategies, as well as indirect comparisons constructed from trials that had one treatment in common. We performed the indirect comparisons using the Canadian Agency for Drugs and Technologies in Health (CADTH) Indirect Treatment Comparisons software, version 1.0 (CADTH, Ottawa, ON, Canada) (17).

For safety, we also used the Bayesian method under the logit random effects consistency model with the assumption of a homogeneous variance for the specified random effect fitted to perform the MTC on the log-odds scale. The default values of various options in the GPU-Enabled Many-Task Computing (GeMTC) package were applied for the required Bayesian computations in $\mathrm{R}$. The potential inconsistency between direct and indirect comparisons could not be detected, as the inconsistency degree of freedom was 0 in our study. We used the GeMTC R package, version 0.2 (February 19, 2013) software, version 2.15.2 (R Foundation for Statistical Computing, Vienna, Austria) (18).

\section{Results}

Results of literature search. Fig. 1 depicts the flow diagram of the systematic literature search and selection of RCTs. The systematic literature search identified 103 abstracts. A total of 81 articles were excluded, as they were not RCTs or did not investigate at least one of our targeted drugs. After reviewing 22 full-length articles, only 7 RCTs matched the inclusion criteria of this study (19-24). Two studies were head-to-head comparisons of two newer targeted agents.

Characteristics of the selected RCTs. The demographic and clinical characteristics of the 7 selected RCTs are summarized in Table I. Each targeted agent was compared to a placebo in RCTs. All the patients enrolled in the included studies were aged $>18$ years, were diagnosed with metastatic clear cell RCC, had a life expectancy of 12 weeks and had an Eastern Cooperation Oncology Group performance status of 0 or 1 . All the 


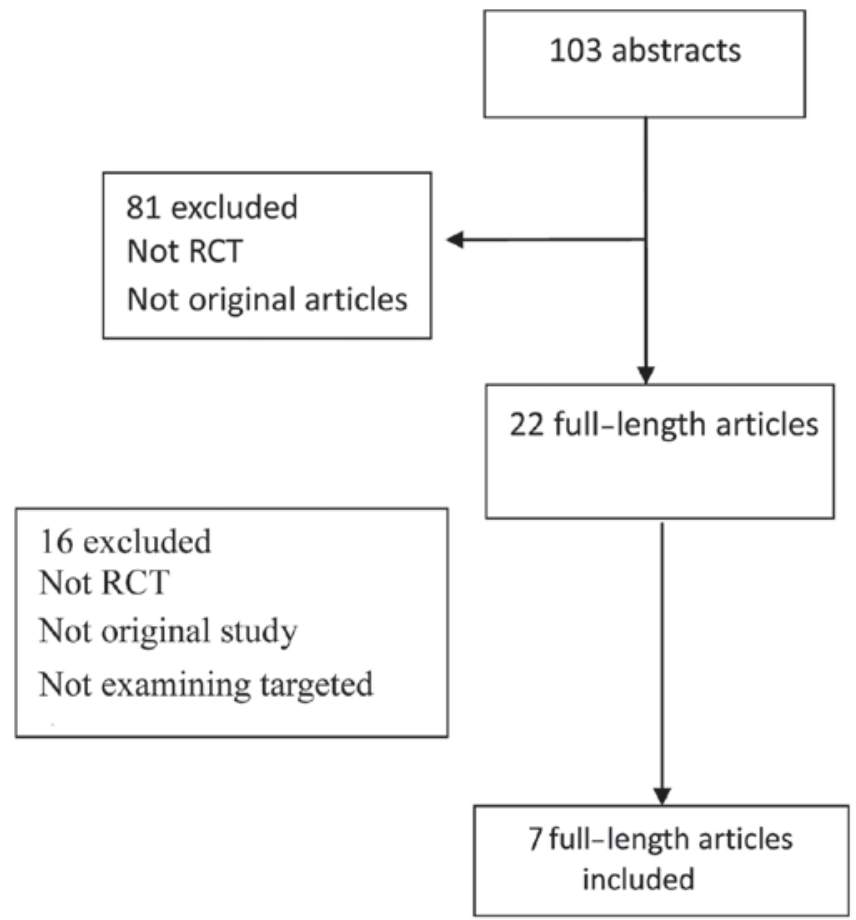

Figure 1. Flow chart of the study selection process. RCT, randomised clinical trial.

patients had previously undergone nephrectomy or received a systematic cytokine-based treatment. The prognostic risks were classified according to the Memorial Sloan-Kettering Cancer Center prognostic score (22).

Quality assessment. The evaluation of the risk of each bias for the 7 selected RCTs is summarized in Fig. 2.

A total of $28.6 \%$ (2/7) of the trials described the method used to generate the allocation sequence in sufficient detail to allow an assessment of this item. A total of $57.1 \%$ (4/7) of the trials had allocation concealment. No studies were completely blinded and the participants were allowed to be open-label when they exhibited progression during treatment. A total of 57.1\% (4/7) of the trials reported blinded outcome assessors. Withdrawal or dropouts were reported in $85.7 \%$ (6/7) of the trials (19-25). All the studies were found to have a low risk of bias for selective outcome reporting. In these studies, all expected outcomes were reported, including important side effects and those outcomes that were pre-specified in Materials and methods. All the included studies reported that adverse events were actively monitored; therefore, the risk of bias was considered to be low.

Direct comparison of treatment effects (targeted agents vs. placebo). The network graph and forest plot were drawn to graphically display the results of the available direct comparisons between treatments (Figs. 3 and 4). The outcome data were extracted from the 7 selected RCTs including a total of 3,390 patients (Table I). The 7 newer targeted agents achieved a statistically significant improvement in PFS compared to placebo, with a non-significant higher risk of adverse events (Fig. 4).

Indirect comparisons of treatment effects. The network graph in Fig. 5 shows the results of our network meta-analysis

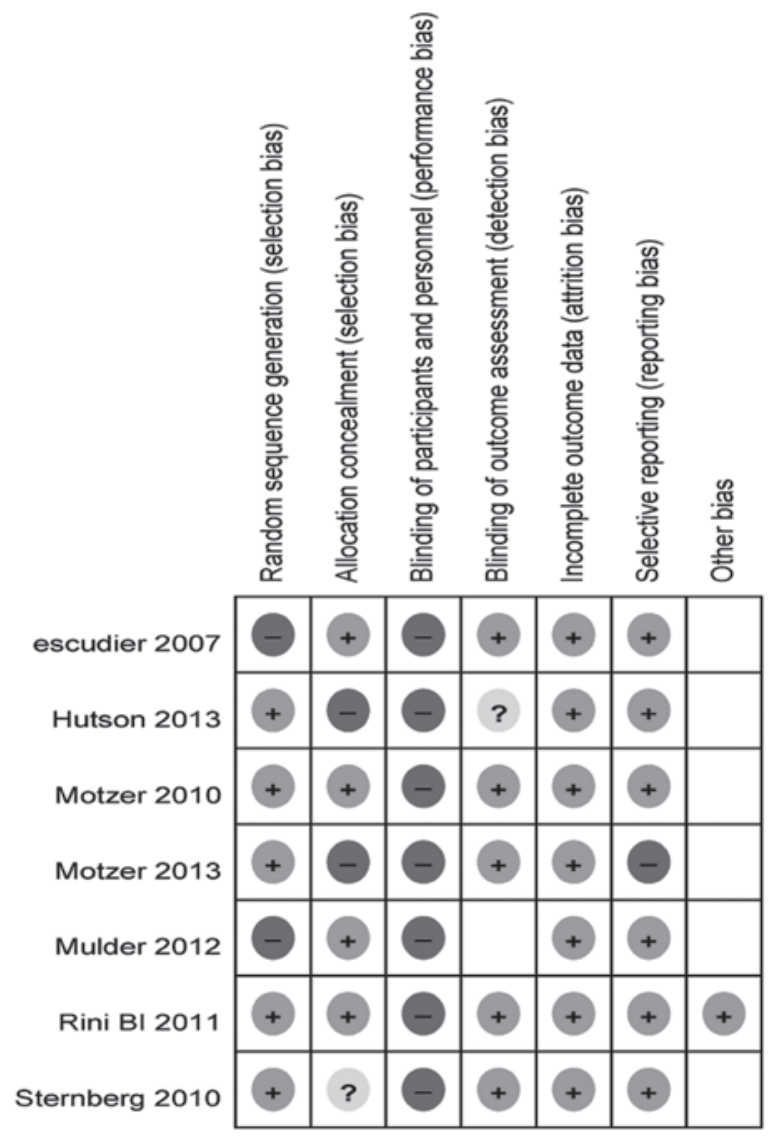

Figure 2. Risk of bias in 7 randomized clinical trials.

for comparisons of efficacy among the 7 newer targeted agents for the treatment of mRCC. Specifically, as listed in the forest plots in Fig. 6, axitinib exhibited significantly improved efficacy compared to panzopanib [HR $=0.64,95 \%$ confidence interval (CI): 0.42-0.98] and sorafenib (HR=0.67, 95\% CI: 0.54-0.81); sunitinib was superior to sorafenib (HR=1/1.63=0.61, 95\% CI: $1 / 2.45-1 / 1.09)$ and temsirolimus (HR $=0.73$, 95\% CI: 0.56-0.96) regarding the efficacy outcome.

We also conducted a network meta-analysis for comparisons of safety among the 7 newer targeted agents, which did not reveal any statistically significant differences in the number of withdrawals of adverse events (Fig. 7). As shown in Fig. 8, the ranking probabilities of the competing treatments and placebo from the network meta-analysis of safety indicated that, of the 7 newer targeted agents, axitinib was likely to be associated with the lowest risk of withdrawals due to adverse events; conversely, cediranib was associated with the highest risk of withdrawals due to adverse events.

\section{Discussion}

The future trend in the treatment of RCC is the introduction of novel agents that may prove more efficacious in the prolongation of PFS and cause fewer adverse events compared to the conventional systematic cytokine therapy. The 7 investigated targeted agents (sorafenib, sunitinib, temsirolimus, everolimus, pazopanib, axitinib and cediranib) have been approved by the Food and Drug Administration in the United States and Europe. The lack of head-to-head studies comparing these targeted 


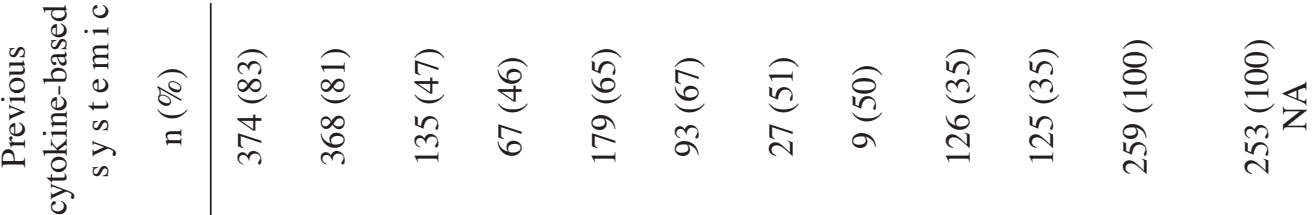

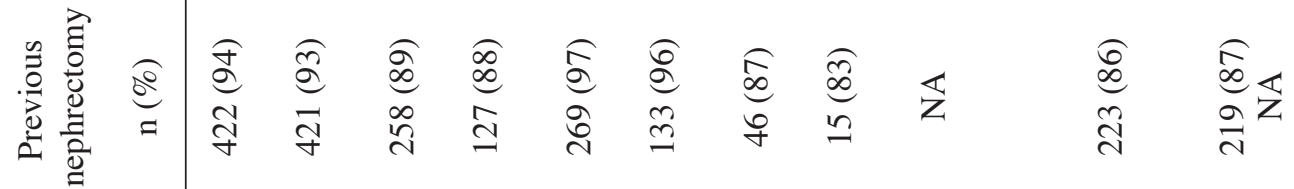

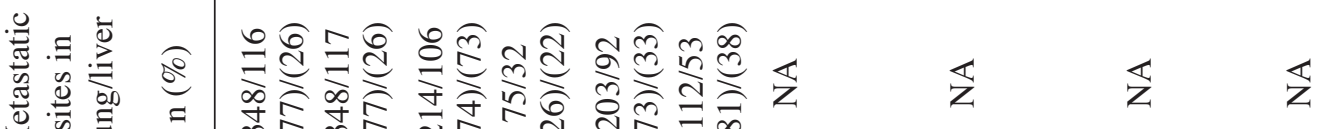

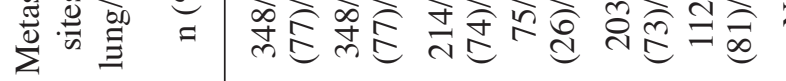

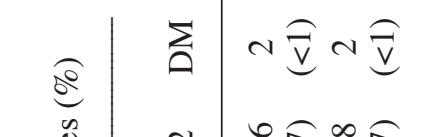

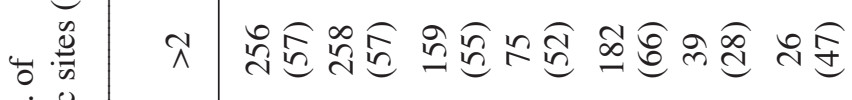

$\dot{\mathrm{z}} \cdot \frac{\mathrm{m}}{\mathrm{n}}$

त

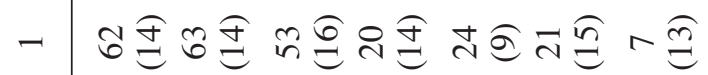

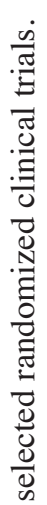

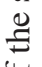

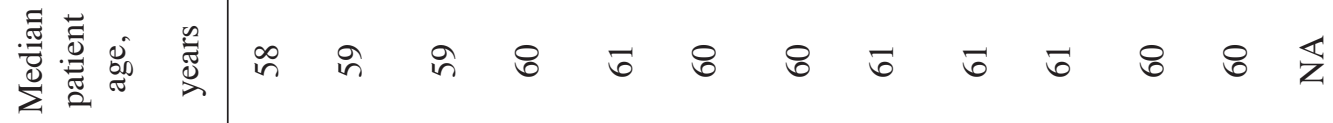

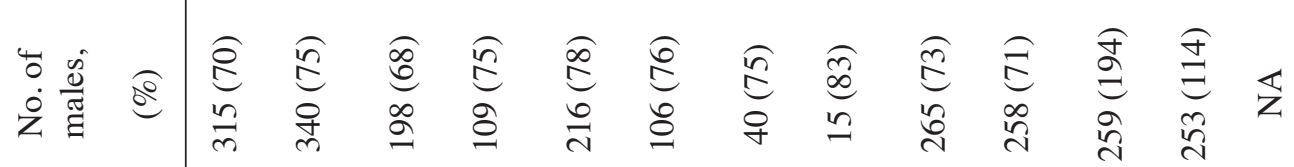

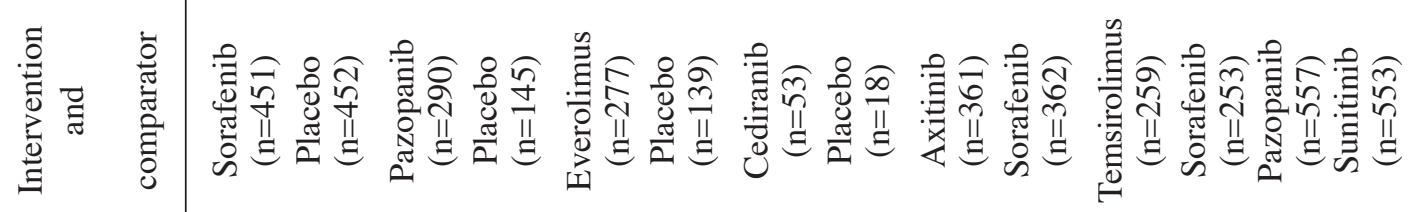

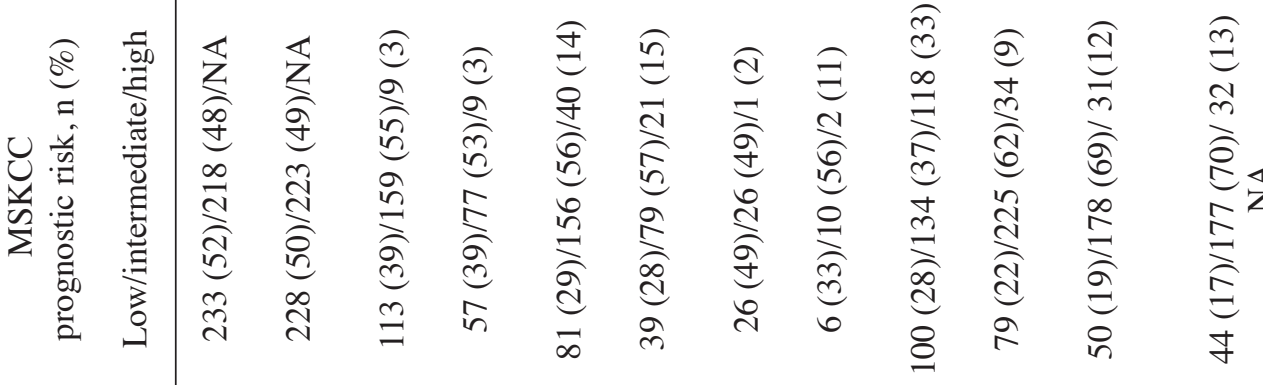

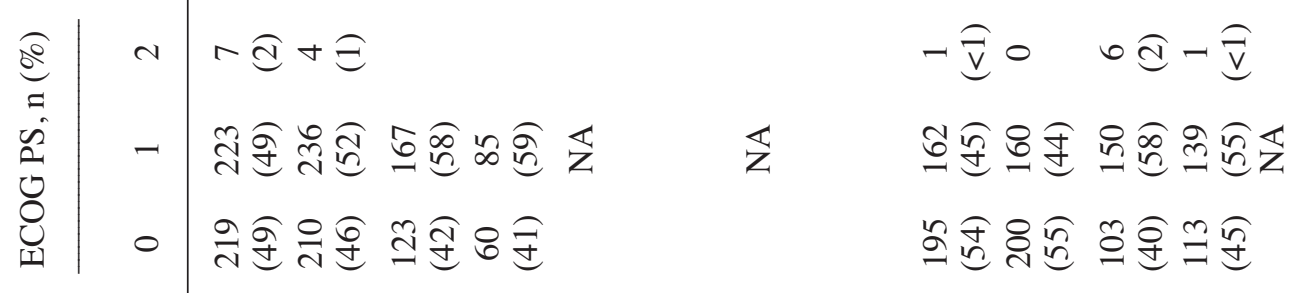

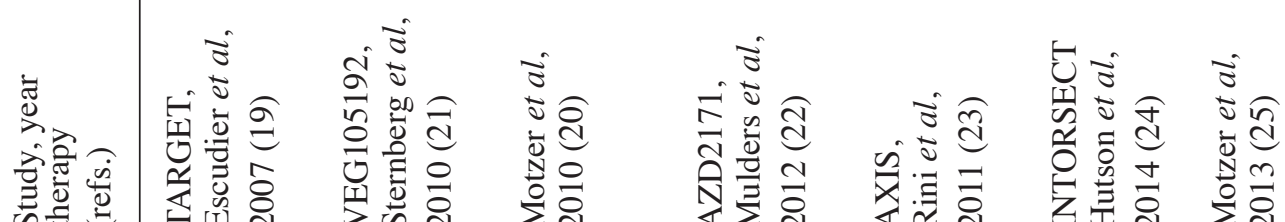




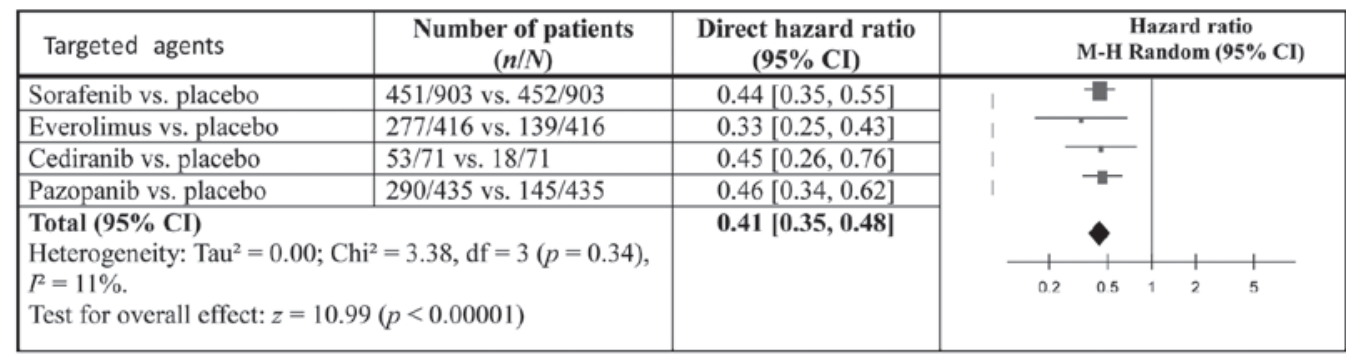

Figure 3. Direct comparison of each targeted agent to placebo/IFN- $\alpha$ for benefit (defined as prolonged progression-free survival). CI, confidence interval.

\begin{tabular}{|c|c|c|c|c|}
\hline Targeted agents & $\begin{array}{l}\text { Number of patients } \\
(\mathrm{n} / \mathrm{N})\end{array}$ & $\begin{array}{l}\text { Direct Odds ratio } \\
\qquad(95 \% \mathrm{Cl})\end{array}$ & \multicolumn{2}{|c|}{$\begin{array}{l}\text { Odds Ratio } \\
\mathrm{M}-\mathrm{H} \text {, random, } 95 \% \mathrm{Cl}\end{array}$} \\
\hline Sorafenib vs. placebo & $18 / 451$ vs. $17 / 451$ & $1.06[0.54,2.09]$ & \multirow{2}{*}{\multicolumn{2}{|c|}{$\rightarrow \longrightarrow$}} \\
\hline Everolimus vs. placebo & $36 / 274$ vs. $2 / 137$ & $10.21[2.42,43.07]$ & & \\
\hline Cediranib vs. placebo & 6/53 vs. $2 / 18$ & $1.02[0.19,5.58]$ & \multirow{2}{*}{\multicolumn{2}{|c|}{--}} \\
\hline Pazopanib vs. placebo & $41 / 290$ vs. $5 / 145$ & $4.61[1.78,11.94]$ & & \\
\hline \multicolumn{2}{|l|}{ Total $(95 \% \mathrm{Cl})$} & $2.61[0.84,8.17]$ & & \\
\hline \multicolumn{2}{|c|}{$\begin{array}{l}\text { Heterogeneity: } \mathrm{Tau}^{2}=0.98 ; \mathrm{Chi}^{2}=12.76, \mathrm{df}=3(\mathrm{P}=0.005) \\
\left.\right|^{2}=76 \%\end{array}$} & & \multicolumn{2}{|c|}{$\begin{array}{l}1.005 \\
\text { Favours placebo }\end{array}$} \\
\hline
\end{tabular}

Figure 4. Direct comparison of each target agent to placebo for safety (determined by the number of withdrawals due to adverse events). CI, confidence interval.

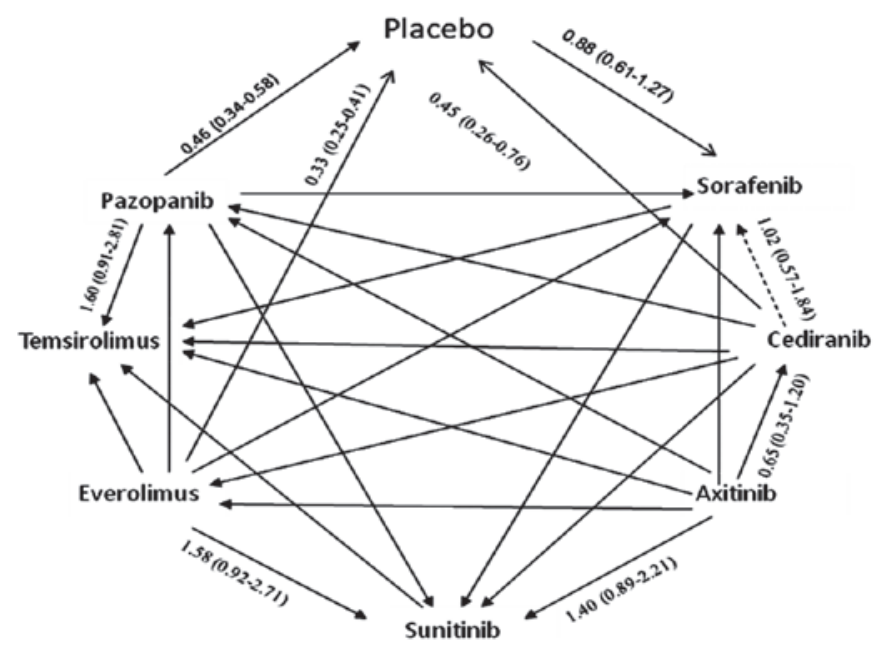

Figure 5. Network of randomized clinical trials of 7 targeted agents for metastatic renal cell carcinoma. For each pairwise comparison, the arrowhead points to the targeted agents for efficacy. The summary hazard ratio and $95 \%$ confidence interval for comparison are shown.

agents in either the first- or second-line treatment is the major weak point in evidence-based medicine. Three head-to-head trials comparing two targeted agents were recently completed; however, only the AXIS study has published its results (23). More direct head-to-head studies comparing two targeted agents are likely to be developed in the future based on the current trial's results. Therefore, we performed this network meta-analysis on efficacy and safety by using the available data from clinical trials in order to provide patients and clinical practitioners with currently available information and enable the selection of an effective and safe treatment.

The results of this network indirect analysis suggest that axitinib is a more suitable targeted therapy option to prolong
PFS after failure of the first-line treatment of mRCC. Additionally, the findings of this analysis also suggested that sunitinib is superior to sorafenib. This finding is consistent with those from two recently published indirect comparison studies, which indicated that sunitinib was superior to sorafenib $(\mathrm{HR}=0.58$, 95\% CI: 0.38-0.86, $\mathrm{P}<0.001$; and $\mathrm{HR}=0.47,95 \%$ CI: $0.316-0.713$, $\mathrm{P}<0.001)$ in the treatment of $\operatorname{mRCC}(26,27)$. The results of our meta-analysis also indicated that axitinib exhibits a higher efficacy and safety compared to sorafenib and pazopanib in patients who previously received systematic treatment. This finding is also in line with a recent systematic review and meta-analysis for second-line treatments in the management of advanced RCC (28). The authors of that study reported that axitinib was superior to placebo $(\mathrm{HR}=0.25,95 \% \mathrm{CI}$ : $0.17-0.38)$ or sorafenib $(\mathrm{HR}=0.46,95 \% \mathrm{CI}: 0.32-0.68)$ and pazapanib $(\mathrm{HR}=0.47$, 95\% CI: 0.26-0.85) in prolonging PFS.

Targeted agents are associated with a distinct pattern of adverse events in $\mathrm{mRCC}$, with the specific profiles and relative severities varying by agent. The development of practical adverse event management techniques to enable patients to achieve the greatest benefit from treatment is an increasing concern. In this study, we demonstrated that axitinib is the least likely to be associated with adverse events among the 7 newer targeted agents, a finding supported by the recent published AXIS study. That study reported that axitinib was better tolerated compared to other targeted agents, with a lower incidence of the adverse events often seen with the currently approved targeted agents, such as erythrodysaesthesia, cutaneous toxicities and myelosuppression (23).

The cost of targeted therapy regimens is on the increase as targeted agents are constantly developed. The incremental cost for a life-year gained is 70,000 pounds for sorafenib (29) 67,000 USD for sunitinib and 90,000 pounds for temsirolimus (30). The cost of newer targeted agents is also increasing in our 


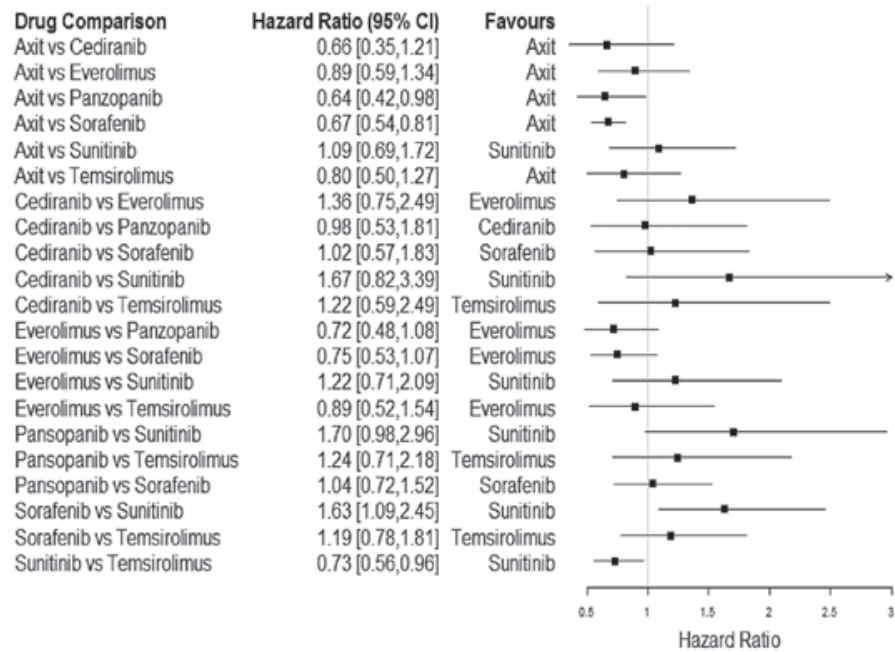

Figure 6. Indirect comparison of each targeted agent to each other for benefit defined as prolonged PFS). A benefit from the targeted agent listed as favours. $\mathrm{CI}=$ confidence interval.)

$\begin{array}{lc}\text { Drug comparison } & \text { Odds ratio }(95 \% \mathrm{CI}) \\ \text { Axitinib vs . cediranib } & 0.56[0.10,3.06] \\ \text { Axitinib vs . everolimus } & 0.14[0.05,0.38] \\ \text { Axitinib vs. pazopanib } & 0.14[0.04,0.49] \\ \text { Axitinib vs. sorafenib } & 0.45[0.24,0.84] \\ \text { Axitinib vs. sunitinib } & 0.44[0.16,1.22] \\ \text { Axitinib vs. temsirolimus } & 0.69[0.18,2.60] \\ \text { Cediranib vs . everolimus } & 0.25[0.05,1.41] \\ \text { Cediranib vs. pazopanib } & 0.25[0.04,1.56] \\ \text { Cediranib vs. sorafenib } & 0.81[0.17,3.86] \\ \text { Cediranib vs. sunitinib } & 0.78[0.14,4.46] \\ \text { Cediranib vs . temsirolimus } & 1.23[0.18,8.51] \\ \text { Everolimus vs . temsirolimus } & 4.81[1.25,18.51] \\ \text { Everolimus vs. pazopanib } & 0.97[0.29,3.24] \\ \text { Everolimus vs . sorafenib } & 3.17[1.57,6.40] \\ \text { Everolimus vs. sunitinib } & 3.06[1.06,8.83] \\ \text { Pazopanib vs . sorafenib } & 3.25[1.20,8.80] \\ \text { Pazopanib vs. sunitinib } & 0.96[0.44,2.09] \\ \text { Pazopanib vs. temsirolimus } & 4.94[1.08,22.60] \\ \text { Sorafenib vs. sunitinib } & 0.96[0.44,2.11] \\ \text { Sorafenib vs . temsirolimus } & 1.51[0.48,4.79] \\ \text { Sunitinib vs . temsirolimus } & 0.39[0.15,0.98]\end{array}$

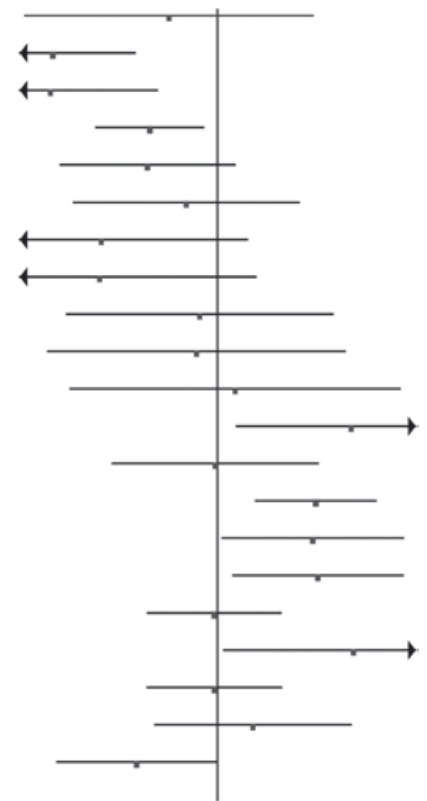

Figure 7. Indirect comparison of each targeted agent to each of the other agents for safety (determined by the number of withdrawals due to adverse events). A value of $>1.0$ indicated a safety concern from the compared targeted agents. CI, confidence interval.
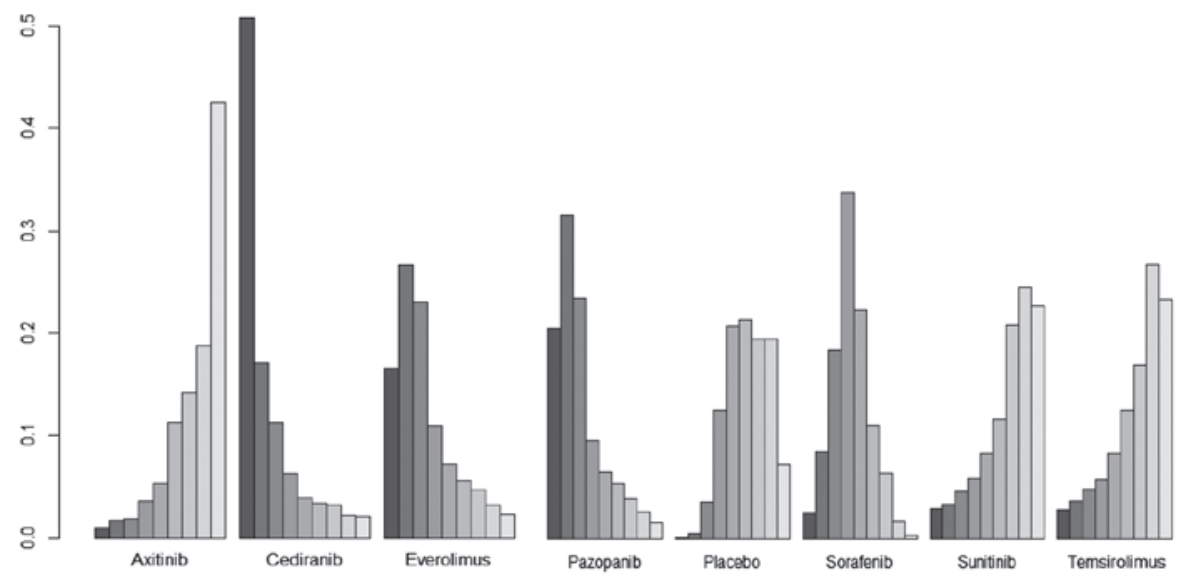

Figure 8. Ranking probabilities of 7 targeted agents. The size of the bar corresponds to the probability of adverse events in each treatment. 
country. Therefore, we must select the most cost-effective and best tolerated drugs to treat patients in order to conserve the limited medical resources.

There are some strengths and limitations to consider when interpreting our analysis. The strengths of our analysis include synthesizing data and comparing the efficacy and safety of all 7 newer targeted agents using the relatively new approach of network meta-analysis. We systematically extracted data from the published RCTs and investigated the risk of bias of the included studies in accordance with the Cochrane Handbook published in 2011 (31). In addition, patients with mRCC did not undergo previous interventions other than systematic therapy and no study assessed the relative effectiveness of each new targeted agent. Our study aimed to provide preliminary comparative results of these newer targeted agents to patients and clinical practitioners to enable selection of the optimal targeted agents.

There were some limitations to our meta-analysis. First, those 7 targeted agents have been available over a relatively short period of time; thus, the duration of the trials was not sufficient to assess the long-term benefits and risks. Moreover, some heterogeneity and reporting bias existed in the identified RCTs, which is likely to affect the results of this study. Second, unpublished data were excluded in the search strategy; therefore, publication bias may exist in this analysis. Third, we used the GRADE method to assess and grade the quality of the evidence; however, the grading process entirely depends on the reviewer's personal judgment and, therefore, the quality may be underestimated.

In conclusion, the results of this network analysis indicated that sunitinib and axitinib may offer some clinical benefit superior to that of sorafenib, pazopanib and temsirolimus. Axitinib was associated with the lowest risk of withdrawals due to adverse events. This summary of evidence may provide preliminary data regarding the efficacy and safety of targeted agents to physicians and patients enabling the design of evidence-based treatment strategies for mRCC.

\section{References}

1. Ferlay J, Shin HR, Bray F, Forman D, Mathers C and Parkin DM: Estimates of worldwide burden of cancer in 2008: GLOBOCAN 2008. Int J Cancer 127: 2893-2917, 2010.

2. Jemal A, Thun MJ, Ries LA, et al: Annual report to the nation on the status of cancer, 1975-2005, featuring trends in lung cancer, tobacco use, and tobacco control. J Natl Cancer Inst 100: 1672-1694, 2008.

3. Mathew A, Devesa SS, Fraumeni JF Jr and Chow WH: Global increases in kidney cancer incidence, 1973-1992. Eur J Cancer Prev 11: 171-178, 2002.

4. Jemal A, Siegel R, Xu J and Ward E: Cancer statistics, 2010. CA Cancer J Clin 60: 277-300, 2010.

5. Taiwan Cancer Registry:http://tcr.cph.ntu.edu.tw/main. php?Page=N1. Accessed June 21, 2012.

6. Gupta K, Miller JD, Li JZ, Russell MW and Charbonneau C: Epidemiologic and socioeconomic burden of metastatic renal cell carcinoma (mRCC): a literature review. Cancer Treat Rev 34: 193-205, 2008

7. Athar U and Gentile TC: Treatment options for metastatic renal cell carcinoma: a review. Can J Urol 15: 3954-3966, 2008.

8. Shih YC, Chien CR, Xu Y, Pan IW, Smith GL and Buchholz TA: Economic burden of renal cell carcinoma: Part I - an updated review. Pharmacoeconomics 29: 315-329, 2011.

9. Finley DS, Pantuck AJ and Belldegrun AS: Tumor biology and prognostic factors in renal cell carcinoma. Oncologist 16 (Suppl 2): 4-13, 2011.
10. Schünemann H, Brozek J and Oxman A (eds): GRADE handbook for grading quality of evidence and strength of recommendation, version 3.2 (updated March 2009). The GRADE Working Group. http://ims.cochrane.org/revman/gradepro. Accessed June 21, 2012.

11. Higgins JPT and Green S (eds): Cochrane Handbook for Systematic Reviews of Interventions, version 5.0.0. The Cochrane Collaboration, 2008.

12. Lumley T: Network meta-analysis for indirect treatment comparisons. Stat Med 21: 2313-2324, 2002.

13. Lu G and Ades AE: Combination of direct and indirect evidence in mixed treatment comparisons. Stat Med 23: 3105-3124, 2004.

14. Review Manager (RevMan) computer program, version 5.2. Copenhagen: The Nordic Cochrane Centre, The Cochrane Collaboration, 2013.

15. Bucher HC, Guyatt GH, Griffith LE and Walter SD: The results of direct and indirect treatment comparisons in meta-analysis of randomized controlled trials. J Clin Epidemiol 50: 683-691, 1997.

16. Caldwell DM, Ades AE and Higgins JP: Simultaneous comparison of multiple treatments: combining direct and indirect evidence. BMJ 331: 897-900, 2005.

17. Wells GA, Sultan SA, Chen L, Khan M and Coyle D: Indirect Treatment Comparisons (ITC) computer program, version 1.0. Ottawa: Canadian Agency for Drugs and Technologies in Health, 2009.

18. R Development Core Team (2011): R: A Language and Environment for Statistical Computing. R Foundation for Statistical Computing, Vienna, Austria. http://www.R-project.org/. Accessed August 14, 2013.

19. Escudier B, Eisen T, Stadler WM, et al; TARGET Study Group: Sorafenib in advanced clear-cell renal-cell carcinoma. N Engl J Med 356: 125-134, 2007.

20. Motzer RJ, Escudier B, Oudard S, et al; RECORD-1 Study Group: Phase 3 trial of everolimus for metastatic renal cell carcinoma: final results and analysis of prognostic factors. Cancer 116: 4256-4265, 2010.

21. Sternberg CN, Davis ID, Mardiak J, et al: Pazopanib in locally advanced or metastatic renal cell carcinoma: results of a randomized phase III trial. J Clin Oncol 28: 1061-1068, 2010.

22. Mulders $\mathrm{P}$, Hawkins R, Nathan $\mathrm{P}$, et al: Cediranib monotherapy in patients with advanced renal cell carcinoma: results of a randomised phase II study. Eur J Cancer 48: 527-537, 2012.

23. Rini BI, Escudier B, Tomczak P, Kaprin A, Szczylik C, Hutson TE, et al: Comparative effectiveness of axitinib versus sorafenib in advanced renal cell carcinoma (AXIS): a randomised phase 3 trial. Lancet 378: 1931-1939, 2011.

24. Hutson TE, Escudier B, Esteban E, et al: Randomized phase III trial of temsirolimus versus sorafenib as second-line therapy after sunitinib in patients with metastatic renal cell carcinoma. J Clin Oncol 32: 760-767, 2014.

25. Motzer RJ, Hutson TE, Cella D, et al: Pazopanib versus sunitinib in metastatic renal-cell carcinoma.N Engl J Med 369: 722-731, 2013.

26. Mills EJ, Rachlis B, O'Regan C, et al: Metastatic renal cell cancer treatments: an indirect comparison meta-analysis. BMC Cancer 9: 34, 2009.

27. Leung HW and Chan AL: Multikinase inhibitors in metastatic renal cell carcinoma: indirect comparison meta-analysis. Clin Ther 33: 708-716, 2011.

28. Larkin J, Paine A, Tumur I, Cappelleri JC, Healey PJ Sr, Foley G, Mitchell S, Kroes M and Chen C: Second-line treatments for the management of advanced renal cell carcinoma: systematic review and meta-analysis. Expert Opin Pharmacother 14: 27-39, 2013.

29. Hoyle M, Green C, Thompson-Coon J, Liu Z, Welch K, Moxham T and Stein K: Cost-effectiveness of sorafenib for second-line treatment of advanced renal cell carcinoma. Value Health 13: 55-60, 2010.

30. Hoyle M, Green C, Thompson-Coon J, Liu Z, Welch K, Moxham T and Stein K: Cost-effectiveness of temsirolimus for first-line treatment of advanced renal cell carcinoma. Value Health 13: 61-68, 2010.

31. Higgins JPT and Green S (eds): Cochrane Handbook for Systematic Reviews of Interventions, version 5.1.0. The Cochrane Collaboration, 2011. 